\title{
Bioinformatic identification of Mycobacterium tuberculosis proteins likely to target host cell mitochondria: virulence factors?
}

\author{
María Maximina Bertha Moreno-Altamirano', Iris Selene Paredes-González ${ }^{1}$, Clara Espitia², \\ Mauricio Santiago-Maldonado', Rogelio Hernández-Pando ${ }^{3}$ and Francisco Javier Sánchez-García ${ }^{*}$
}

\begin{abstract}
Background: M. tuberculosis infection either induces or inhibits host cell death, depending on the bacterial strain and the cell microenvironment. There is evidence suggesting a role for mitochondria in these processes.

On the other hand, it has been shown that several bacterial proteins are able to target mitochondria, playing a critical role in bacterial pathogenesis and modulation of cell death. However, mycobacteria-derived proteins able to target host cell mitochondria are less studied.

Results: A bioinformaic analysis based on available genomic sequences of the common laboratory virulent reference strain Mycobacterium tuberculosis H37Rv, the avirulent strain H37Ra, the clinical isolate CDC1551, and M. bovis BCG Pasteur strain 1173P2, as well as of suitable bioinformatic tools (MitoProt II, PSORT II, and SignalP) for the in silico search for proteins likely to be secreted by mycobacteria that could target host cell mitochondria, showed that at least $19 \mathrm{M}$. tuberculosis proteins could possibly target host cell mitochondria. We experimentally tested this bioinformatic prediction on four M. tuberculosis recombinant proteins chosen from this list of 19 proteins (p27, PE_PGRS1, PE_PGRS33, and MT_1866). Confocal microscopy analyses showed that p27, and PE_PGRS33 proteins colocalize with mitochondria.

Conclusions: Based on the bioinformatic analysis of whole $M$. tuberculosis genome sequences, we propose that at least 19 out of 4,246 M. tuberculosis predicted proteins would be able to target host cell mitochondria and, in turn, control mitochondrial physiology. Interestingly, such a list of 19 proteins includes five members of a mycobacteria specific family of proteins (PE/PE_PGRS) thought to be virulence factors, and p27, a well known virulence factor. P27, and PE_PGRS33 proteins experimentally showed to target mitochondria in $\mathrm{J774}$ cells. Our results suggest a link between mitochondrial targeting of $M$. tuberculosis proteins and virulence.
\end{abstract}

Keywords: Mycobacterium tuberculosis, Mitochondrial targeting, Virulence

\section{Background}

In spite of the huge efforts to overcome the burden of tuberculosis (TB), nearly 10 million incident cases of TB cases, the death of 1.1 million HIV-negative TB patients and an additional 0.35 million deaths from HIV-associated TB are reported each year. Unfortunately, the selection and spread of multidrug-resistant (MDR) Mycobacterium tuberculosis strains worsen the

\footnotetext{
* Correspondence: fsanchez_1@yahoo.co.uk

'Laboratorio de Inmunorregulación, Departamento de Inmunología, Escuela Nacional de Ciencias Biológicas, Instituto Politécnico Nacional, Carpio y Plan de Ayala, Col. Sto. Tomás, México D.F, México

Full list of author information is available at the end of the article
}

scenario, since an estimated 0.65 million cases of MDRTB were documented for the year 2010 [1]. Clearly, in addition to the improvement of human population welfare, the development of new vaccines, early diagnosis tests, and pharmacological treatments, a precise knowledge on mycobacteria-host cell interactions is also a requirement for the successful control of TB.

In this regard, some bacterial pathogenicity factors have been shown to contain $\mathrm{N}$-terminal mitochondrial targeting signals $[2,3]$ and a diverse array of bacterial proteins including some bacterial toxins from enteropathogenic E.coli. Salmonella spp, N. gonorrhoea,

\section{() Biomed Central}

(c) 2012 Moreno-Altamirano et al.; licensee BioMed Central Ltd. This is an Open Access article distributed under the terms of the Creative Commons Attribution License (http://creativecommons.org/licenses/by/2.0), which permits unrestricted use, distribution, and reproduction in any medium, provided the original work is properly cited. 
N. meningitides, A. baumanii, H. pylori, S. aureus, S. pneumoniae, $C$. sordelli, and $C$. difficile have been shown to target mitochondria [4].

Two proteins of particular interest are mitochondrialassociated protein (Map, former Orf19) and EspF from enteropathogenic E.coli, which enter the host cell via the type 3 secretion system and colocalize with host mitochondria [2]. It is worth noting that, Map causes alterations, in the form of mitochondrial membrane potential $\left(\Delta \psi_{\mathrm{m}}\right)$ dissipation but apparently it is not responsible for enteropathogenic E.coli-induced host cell apoptosis $[2,4,5]$.

Mycobacterial infection affects mitochondrial function [6-8] and it has been suggested that $\Delta \psi_{\mathrm{m}}$ changes are related to the mycobacterial strain/s virulence $[7,8]$. However, the identity of the molecules responsible for such a response has not been defined. Recently, the $M$. tuberculosis protein PE_PGRS33 was shown to localize within host cell mitochondria, and in doing so, induces host cell apoptosis [9].

Since mitochondria targeting proteins such as Map and EspF (from enteropathogenic E. coli) are secreted proteins, we thought that it is likely that at least some of the mycobacterial proteins that potentially target host cell mitochondria are also secreted proteins. Therefore, we set up a bioinformatic search of $M$. tuberculosis proteins likely to be secreted and to target host cell mitochondria.

The M. tuberculosis genome was first made available in 1998, opening the possibility for in-depth analysis of the possible pathogenic mechanisms involved in the course of mycobacterial infections [10]. Other mycobacterial genomes have been elucidated since, and new bio-informatic resources allow for in silico analysis of their genomes $[11,12]$.

Genome wide analysis predicts that M. tuberculosis H37Rv and CDC1551 contain 3,924 and 4,246 genes, respectively $[10,11]$.

By using freely available data bases and bioinformatic tools, we were able to single out $19 \mathrm{M}$. tuberculosis CDC1551 predicted proteins as likely candidates for targeting mitochondria.

Among these, five PE/PE_PGRS family proteins deserve particular attention. About $10 \%$ of the potential coding capacity of $M$. tuberculosis accounts for two large unrelated gene families encoding the PE and PPE proteins. The names PE and PPE are derived from the motifs Pro-Glu (PE) and Pro-Pro-Glu (PPE), and the largest class of the PE family in M. tuberculosis H37Rv is the PE_PGRS subfamily which consist of proteins with a $\mathrm{PE}$ domain followed by a C-terminal glycine-rich extension encoded by the PGRS motif (polymorphic GCrich repetitive sequence) $[13,14]$. As a family, the PE/ PE_PGRS proteins are polymorphic [10] and account for many of the differences found between the avirulent (H37Ra) and virulent (H37Rv) strains of M. tuberculosis and, in consequence, have been proposed as possible virulence factors [15].

\section{Results}

M. tuberculosis proteins predicted to be secreted and to target host cell mitochondria

The whole 4,246 predicted proteins from the $M$. tuberculosis CDC1551 genome as shown in the JCVI/CMR webpage were analyzed for the presence of mitochondrial targeting sequences by using the MitoProt IIv1.101 algorithm. Although previous analyses using this algorithm on other bacterial proteomes have set the probability limit at 0.700 to consider a protein as likely to target mitochondria [16], we decided for a more stringent analysis and therefore we set the probability limit at 0.8500 that would help to reduce false negatives. As shown in Figure 1, a total of 337 out of 4,246 M. tuberculosis CDC1531 proteins are predicted to have mitochondrial targeting potential. Next we analyzed those 337 proteins by using another mitochondrial targeting predictive algorithm (PSORT II), and found that 136 out of the 337 proteins scored as likely to target host cell mitochondria. Finally, the 136 predicted proteins that scored positive for both predictive algorithms were further analyzed for the probability of harboring secretory signal peptides by using the SignalP 3.0 software. Taken together, these three bioinformatic resources allowed us to identify 19 predicted proteins with the probability of harboring secretory peptide signals as well as mitochondrial targeting signals. Table 1 , lists the identity of the 47 M. tuberculosis predicted proteins (Top-47) with the highest combined MitoProt II and PSORT II probability of targeting mitochondria. Table 2, list the 19 predicted proteins from $M$. tuberculosis CDC1551strain, likely to be secreted by the mycobacteria and to target host cell mitochondria.

\section{PE and PE_PGRS family proteins are among the M. tuberculosis CDC1551 proteins likely to be secreted and targeted to host cell mitochondria}

Table 2 shows that the bioinformatic procedure used in this work selected 19 out of 4,246 predicted proteins from the $M$. tuberculosis CDC1551 strain genome that are predicted to be secreted and targeted to the host cell mitochondria. By looking at the annotated characteristics of those proteins, it is evident that members from the PE and PE_PGRS family proteins are overrepresented $(5 / 19,26.3 \%)$, since the total number of PE and PE_PGRS proteins is estimated to be 94 , by manually counting them from the JCVI/CMR M. tuberculosis CDC1551 list (94/4,246, 2.21\%). Interestingly, a recent comparative analysis among mycobacterial genomes, 


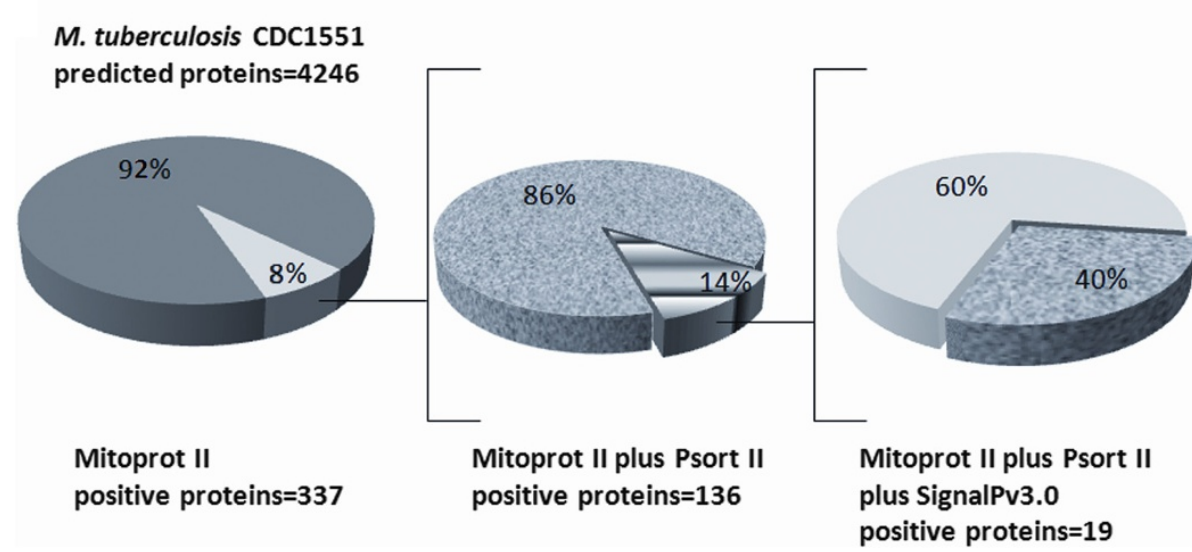

Figure 1 Distribution of predicted $\boldsymbol{M}$. tuberculosis CDC1551 proteins according to their signal peptides. The 4, 246 predicted proteins of M. tuberculosis CDC1551 were analyzed for the presence of mitochondrial targeting sequences by the predictive algorithm MitoProt II, the 337 proteins that scored positive were analyzed by PSORT II, rendering 136 proteins likely to target mitochondria. Finally, these 136 proteins were analyzed for the presence of secretory signal peptides by the SignalP software.

performed in an attempt to identify the mutations that lead to the loss of virulence in the M. tuberculosis H37Ra strain, showed that most mutations were confined to the PE/PPE/ PE_PGRS family genes, highlighting the likely role of those proteins as virulent factors [15]. Accordingly, we performed a search for homologous proteins in M. tuberculosis H37Ra, M. tuberculosis H37Rv, and M. bovis BCG Pasteur $1173 \mathrm{P} 2$ strain and then analyzed those homologous proteins by using MitoProt II, PSORT II, and SignalP algorithms as previously described. Table 3 shows the protein codes for each mycobacterial strain, as well as the possible identity and the scores for the three aforementioned predictive algorithms. These analyses showed that the percentage of similarity among the identified M. tuberculosis CDC1551 strain PE or PE_PGRS proteins and their H37Ra, H37Rv, and BCG Pasteur strain homologous proteins ranged from 53.4\% to $81.9 \%$. The H37Ra (MRA_0115) and BCG (BCG_0142) homologous to CDC1551 MT_0118 protein are predicted as not mitochondrial targeted, whereas the H37Rv (Rv0109) scored positive just as well as MT_0118, and the same holds true for the three homologous proteins to MT_0349, but none of them seem to have secretory signal sequences. All six homologous proteins to CDC1551 MT_0854 and MT_1866 did not score positive for mitochondrial targeting sequences. On the other hand, the three homologous proteins for CDC1551 MT_2690 are predicted to harbor mitochondrial targeting sequences. With the exception of MRA_0344, Rv0335c, and BCG_0374, all other homologous proteins are predicted to harbor secretory signal sequences.

Rv1411c (p27), and Rv1818c (PE_PGRS33) M. tuberculosis proteins target host cell mitochondria

Based on the list of 19 M. tuberculosis CDC1551 proteins likely to target host cell mitochondria (Table 2) as well as on the H37Ra, H37Rv, and BCG Pasteur 1173P2 homologous proteins to M. tuberculosis CDC1551 (Table 3), we selected four proteins to be experimentally tested for targeting mitochondria: MT_1866, and its $\mathrm{H} 37 \mathrm{Rv}$ ortholog Rv1818c (PE_PGRS33); the H37Rv ortholog of MT_0118 (Rv0109 or PE_PGRS1); and the H37Rv ortholog of MT_1455 (Rv1411c or p27). In addition, the M. tuberculosis Rv2031c ( $\alpha$-crystallin) protein that scored negative for mitochondria targeting sequence in a Mitoprot II and PSORT II query, was used as a negative control. Rv1818c has recently shown to target mitochondria [9] and therefore served the purpose of a positive control. All $M$. tuberculosis recombinant proteins harbour a histidine tail that allows detection by means of a FITClabelled anti-His antibody, as described in methods.

Figure 2 shows that p27, and PE_PGRS33 colocalize with mitochondria in J774 cells cultured for $2 \mathrm{~h}$ in the presence of $1 \mu \mathrm{g} / \mathrm{ml}$ of the correspondent recombinant protein. MT_1866 and PE_PGRS1 do not seem to colocalize with mitochondria, whereas $\alpha$-crystallin protein, as expected, do not colacalize with mitochondria.

\section{Discussion}

Macrophages are the main host cells for M. tuberculosis, therefore considerable attention has been devoted to the analysis of the mycobacteria-containing phagosomes [17] and the way by which mycobacteria inhibit their association with lysosomes [18-20], and more recently the host-pathogen cross-talk by transcriptional analyses has gained interest $[21,22]$.

On the other hand, it has been shown that M. tuberculosis infection controls host cells, either by inducing $[23,24]$ or inhibiting their death $[25,26]$, and some $M$. tuberculosis-derived molecules have been associated with those opposing biological activities [27-29]. 
Table 1 Top-47 scored $M$. tuberculosis CDC1551 proteins likely to target mitochondria

\begin{tabular}{|c|c|c|c|}
\hline CDC1551 LOCUS NAME & MITOPROT II SCORE & PSORT II SCORE & PUTATIVE IDENTIFICATION \\
\hline MT_0498 & 0,9908 & 0,9570 & carbon-nitrogen hydrolase family protein \\
\hline MT_2329 & 0,9956 & 0,9130 & hypothetical protein \\
\hline MT_40401 & 0,9717 & 0,9130 & conserved hypothetical protein \\
\hline MT_3169 & 0,9998 & 0,8700 & bah acetyl I-hydrolase \\
\hline MT_1950 & 0,9988 & 0,8700 & conserved hypothetical prorein \\
\hline MT_1950 & 0,9988 & 0,8700 & Mt'M-related protein \\
\hline MT_3682 & 0,9904 & 0,8700 & conserved hypothetical protein \\
\hline MT_1161 & 0,9895 & 0,8700 & DNA-binding protein, putative \\
\hline MT_3067 & 0,9884 & 0,8700 & transcriptional regulator, Ic IR family \\
\hline MT_2080 & 0,9871 & 0,8700 & conserved hypothetical protein \\
\hline MT_3357 & 0,9925 & 0,8260 & hypothetical protein \\
\hline MT_0118 & 0,9796 & 0,8260 & PE_PGRS family protein \\
\hline MT_0503 & 0,9348 & 0,8700 & MagC-related protein \\
\hline MT_1260 & 0,9965 & 0,7830 & hypothetical protein \\
\hline MT_3204 & 0,9956 & 0,7830 & hypothetical protein \\
\hline MT_23652 & 0,9932 & 0,7830 & hypothetical protein \\
\hline MT_0644 & 0,9028 & 0,8700 & hypothetical protein \\
\hline MT_35421 & 0,9887 & 0,7830 & hypothetical protein \\
\hline MT_1210 & 0,9318 & 0,8260 & conserved hypothetical protein \\
\hline MT_1284 & 0,9264 & 0,8260 & hypothetical protein \\
\hline MT_23301 & 0,9603 & 0,7830 & hypothetical protein \\
\hline MT_2838 & 0,9987 & 0,7390 & PPE family protein \\
\hline MT_1457 & 0,9983 & 0,7390 & hypothetical protein \\
\hline MT_1866 & 0,9983 & 0,7390 & PE_PGRS family protein \\
\hline MT_0903 & 0,9983 & 0,7390 & hypothetical protein \\
\hline MT_3166 & 0,8710 & 0,8260 & hypothetical protein \\
\hline MT_07241 & 0,9573 & 0,7390 & hypothetical protein \\
\hline MT_0745 & 0,9982 & 0,6960 & rplR ribosomal protein L18 \\
\hline MT_39721 & 0,9551 & 0,7390 & hypothetical protein \\
\hline MT_2954 & 0,9967 & 0,6960 & IS1539, resolvase \\
\hline MT_3026 & 0,9960 & 0,6960 & mythyltransferase, putative \\
\hline MT_0443 & 0,9906 & 0,6960 & hypothetical protein \\
\hline MT_0144 & 0,9892 & 0,6960 & p450 heme-thiolate protein \\
\hline MT_07621 & 0,9887 & 0,6960 & hypothetical protein \\
\hline MT_32201 & 0,9434 & 0,7390 & hypothetical protein \\
\hline MT_0572 & 0,9834 & 0,6960 & oxidoreductase, short-chain dehydrogenase/reductase family \\
\hline
\end{tabular}

Mitochondria play a pivotal role in programmed cell death [30], and mitochondrial activity has been shown to be disrupted by mycobacterial infection in a process that appears to be dependent on the strain/s virulence [6-8].

It has been shown that several secreted microbial proteins are transferred to the cell during infection and may target mitochondria, playing an important role in bacterial pathogenicity [4,31]; and more recent observations suggest that the mitochondrial role in the regulation of cellular responses to pathogens may go further than the sole regulation of host cell death [32].
Taking all this into account we wanted to analyze the $M$. tuberculosis genome and their predicted proteome in search of moieties likely to play a mitochondrial-mediated pathogenicity role, by using bioinformatic tools.

Gomez et al. [33] have already identified M. tuberculosis secreted proteins by performing a bioinformatic search on the 3,924 initially predicted proteins from the $M$. tuberculosis H37Rv genome [10].

Here, we used a different approach. The whole 4,246 predicted proteins from the $M$. tuberculosis CDC1551 genome [11] were first analyzed for the presence of 


\begin{tabular}{ll} 
Table 2 M. tuberculosis CDC1551 proteins predicted to be \\
secreted and targeted to host cell mitochondria \\
\hline CDC1551 & PUTATIVE IDENTITY \\
\hline MT_0068 & mitR protein \\
\hline MT_0118 & virulence factor mce family protein \\
\hline MT_0349 & PE family protein \\
\hline MT_1548 & Conserved hypothetical protein \\
\hline MT_0824 & Hypothetical protein \\
\hline MT_0854 & PE_PGRS family protein \\
\hline MT_1105 & Hypothetical protein \\
\hline MT_1210 & Conserved hypothetical protein \\
\hline MT_1216 & Hypothetical protein \\
\hline MT_1934 & Hypothetical protein \\
\hline MT_1455 & lipoprotein, 27kDA \\
\hline MT_1866 & PE_PGRS family protein \\
\hline MT_1950 & Conserved hypothetical protein \\
\hline MT_2662 & bacterial extracellular, solute-binding protein \\
\hline MT_2690 & PE-PGRS family protein \\
\hline MT_3169 & bac acetyl-hydrolase \\
\hline MT_3367 & Conserved hypothetical protein \\
\hline MT_2662 & bacterial extracellular, solute-binding protein \\
\hline MT_2690 & PE-PGRS family protein \\
\hline MT_3169 & bah acetyl-hydrolase \\
\hline MT_3367 & Conserved hypothetical protein \\
\hline MT_3413 & \\
\hline &
\end{tabular}

mitochondrial targeting sequences by using MitoProt II-v1.101 and PSORT II algorithms. The CDC1551 strain was chosen on the basis of it being isolated from a relatively recent tuberculosis outbreak.

The MitoProt II analysis we performed was more stringent than similar searches on other bacterial proteomes were authors concluded that at least $5 \%$ of proteins from E.coli are predisposed for targeting mitochondria [16]. In this work, by using two predictive algorithms, it was found that, in M. tuberculosis CDC1551, 136 out of 4,246 (3.2\%) proteins are likely to target mitochondria. Mitoprot IIV1.101 has a level of accuracy of 94.76\%-97.54\% for successfully predicting mitochondrial proteins and $76.79 \%-$ $87.92 \%$ for successfully predicting non-mitochondrial proteins in a protein training group [34], and PSORT II has an accuracy of approximately $60 \%-86 \%$ [35].

The 136 predicted proteins likely to target mitochondria were further analyzed for the presence of secretory signal peptides by using the SignalP 3.0 hidden Markov model, which, incidentally, has recently been reported to outperform other similar tools in predicting M. tuberculosis secreted proteins with a level of accuracy of $100 \%$, as it predicted no false positives or false negatives in a negative set $(n=61)$, and in a positive set $(n=57)$ of mycobacterial proteins, respectively. In addition, this algorithm showed
78.9\% accuracy in predicting the actual cleavage site [36]. Their analysis predicted that between $7.8 \%$ and $10.5 \%$ of the proteins in the proteomes of different mycobacteria (including the CDC1551 strain) are secreted proteins. Our SignalP v3.00 hidden Markov model search predicted that 19 out of the 136 proteins likely to target mitochondria are also secreted proteins, i.e., $13.9 \%$.

In their analysis, Gomez et al. [33] excluded $47 \mathrm{PE} /$ PPE proteins out of the top M. tuberculosis 208 proteins predicted to be secretory, arguing that biased amino acid composition and a repetitive primary sequence could lead to unreliable results in sequence analyses. We did not exclude these proteins from our analysis and we found that some PE_PGRS proteins are not predicted to harbour mitochondrial targeting or secretory signals (data not shown); thus, suggesting that the amino acid composition of these proteins did not introduce any bias in our bioinformatic output. In addition, it has recently been shown that some PE_PGRS and PPE proteins are secreted by at least one mycobacterial species (M. marinum) and that those proteins are transported via the type VII secretion system ESX-5 [37].

Interestingly, among the $19 \mathrm{CDC} 1551$ proteins likely to be secreted and to targeted mitochondria, 5 belong to the PE/PE_PGRS protein family (Table 2).

About $10 \%$ of the coding capacity of the genome is devoted to two large unrelated families of acidic, glycine-rich proteins, the PE (Pro-Glu) and PPE (ProPro-Glu) families, often based on multiple copies of the polymorphic repetitive sequence referred to as PGRSs (polymorphic GC-rich repetitive sequences). It has been suggested that these protein families could be of immunological importance [10], and a possible source of antigenic variability that would help $M$. tuberculosis to evade the host immune system during infection [14]. In addition, by using a computational strategy based on phylogenetic profiling and comparative proteomic analysis, Meszaros et al. [38] have proposed that M. tuberculosis $\mathrm{PE} / \mathrm{PPE}$ proteins can be considered as potential targets for drug design. Although the biological function of the PE proteins is still under study, the PE_PGRS member annotated as Rv1759c has been characterized as fibronectinbinding protein [39] and a genetic screen of M. bovis BCG Pasteur mutagenized with the transposon Tn5367, identified a gene, identical to the Rv1818c gene of $M$. tuberculosis, encoding a PE_PGRS protein that influences the interactions of mycobacteria with macrophages [40]. Rv1818c has also been implied in T lymphocytes apoptosis [41], and it has been shown that most genetic differences between $M$. tuberculosis H37Rv and H37Ra strains, likely to function as virulence factors are in the PE/PPE/ PE_PGRS family genes [15].

The availability of suitable bioinformatic tools allowed us to single out $19 \mathrm{M}$. tuberculosis proteins as likely to 
Table 3 Comparative analysis of some M. tuberculosis CDC1551 strain PE and PE_PGRS proteins with their H37Ra, H37Rv and BCG Pasteur strain orthologes

\begin{tabular}{|c|c|c|c|c|}
\hline & CDC 1551 & H37Ra & H3TRv & BCG Pasterur 1173P2 \\
\hline & MT_0118 & MRA_0115 & Rv0109 & BCG_0142 \\
\hline Putative identity & PE-PGRS family protein & & & \\
\hline$\%$ Similarity & & 81.4 & 81.4 & 81.4 \\
\hline \multicolumn{5}{|l|}{ MITOPROT II } \\
\hline Score & 0.9796 & 0.1144 & 0.9799 & 0.1144 \\
\hline PSORT II Score & $82.6 \%$ & $11.1 \%$ & $82.6 \%$ & $11.1 \%$ \\
\hline \multirow[t]{2}{*}{ Signal P Score } & 0.988 & 0.999 & 0.998 & 0.999 \\
\hline & MT_0349 & MRA_0344 & Rv0335c & BCG_0374 \\
\hline Putative identity & PE family protein & & & \\
\hline$\%$ Similarity & & $81.9 \%$ & $81.9 \%$ & $81.3 \%$ \\
\hline \multicolumn{5}{|l|}{ MITOPROT ॥ } \\
\hline Score & 0.9902 & 0.0157 & 0.9746 & 0.0155 \\
\hline PSORT II Score & $73.9 \%$ & $17.4 \%$ & $69.6 \%$ & $21.7 \%$ \\
\hline \multirow[t]{2}{*}{ Signal P Score } & 0.640 & NS & NS & NS \\
\hline & MT_0854 & MRA_0841 & Rv0832 & BCG_0885 \\
\hline Putative identity & PE-PGRS family protein & & & \\
\hline$\%$ Similarity & & $56.2 \%$ & $56.2 \%$ & $56.2 \%$ \\
\hline \multicolumn{5}{|l|}{ MITOPROT ॥ } \\
\hline Score & 0.9326 & 0.4901 & 0.5935 & 0.5738 \\
\hline PSORT II Score & $73.9 \%$ & $4.3 \%$ & $8.7 \%$ & $11.1 \%$ \\
\hline \multirow[t]{2}{*}{ Signal P Score } & 0.999 & 0.998 & 0.993 & 0.998 \\
\hline & MT_1886 & MRA_1830 & Rv1818c & BCG_1853c \\
\hline Putative identity & PE-PGRS Family & & & \\
\hline \% Similarity & & $53.4 \%$ & $53.4 \%$ & $53.4 \%$ \\
\hline \multicolumn{5}{|l|}{ MITOPROT II } \\
\hline Score & 0.9983 & 0.1494 & 0.1494 & 0.1475 \\
\hline PSORT II Score & $73.9 \%$ & $13.0 \%$ & $13.0 \%$ & $13.0 \%$ \\
\hline \multirow[t]{2}{*}{ Signal P Score } & 0.974 & 0.991 & 0.991 & 0.991 \\
\hline & MT_2690 & MRA_2643 & Rv2615c & BCG_1035C \\
\hline Putative identity & PE-PGRS Family & & & \\
\hline$\%$ Similarity & & $79.0 \%$ & $79.0 \%$ & $78.2 \%$ \\
\hline \multicolumn{5}{|l|}{ MITOPROT II } \\
\hline Score & 0.9472 & 0.9472 & 0.8178 & 0.9488 \\
\hline PSORT II Score & $52.2 \%$ & $52.2 \%$ & $13.0 \%$ & $52.2 \%$ \\
\hline Signal P Score & 0.917 & 0.917 & 0.849 & 0.917 \\
\hline
\end{tabular}

be secreted and to target host cell mitochondria, among them five PE/PE_PGRS family protein members. Recently, Cadieux et al. [9] showed that the M. tuberculosis PE_PGRS33 protein (encoded by the Rv1818c gene) colocalize with mitochondria when expressed in human rhabdomyosarcoma (RD) cells, and mitochondrial localization of PE_PGRS33 protein was followed by induction of cell death [9]. This finding is somewhat in contrast with our in silico analysis, since PE_PGRS33 (RV1818c) is not predicted to harbour a mitochondrial targeting sequence by MitoProt or PSORT II (Table 3) Moreover, the $M$. tuberculosis CDC1551 protein $\mathrm{MT}_{-}$ 1866 which is the ortholog of $\mathrm{Rv} 1818 \mathrm{c}$ is predicted to harbour a mitochondria targeting sequence (Table 3) and experimentally failed to do so (Figure 2). A possible explanation for this is that PE_PGRS proteins are unreliably alignable or differences in the annotation for the strain CDC1551 as compared for the H37Rv stain of $M$. tuberculosis. In addition the similarity score between RV1818c and MT_1866 is just 53.4\%, a very low score 


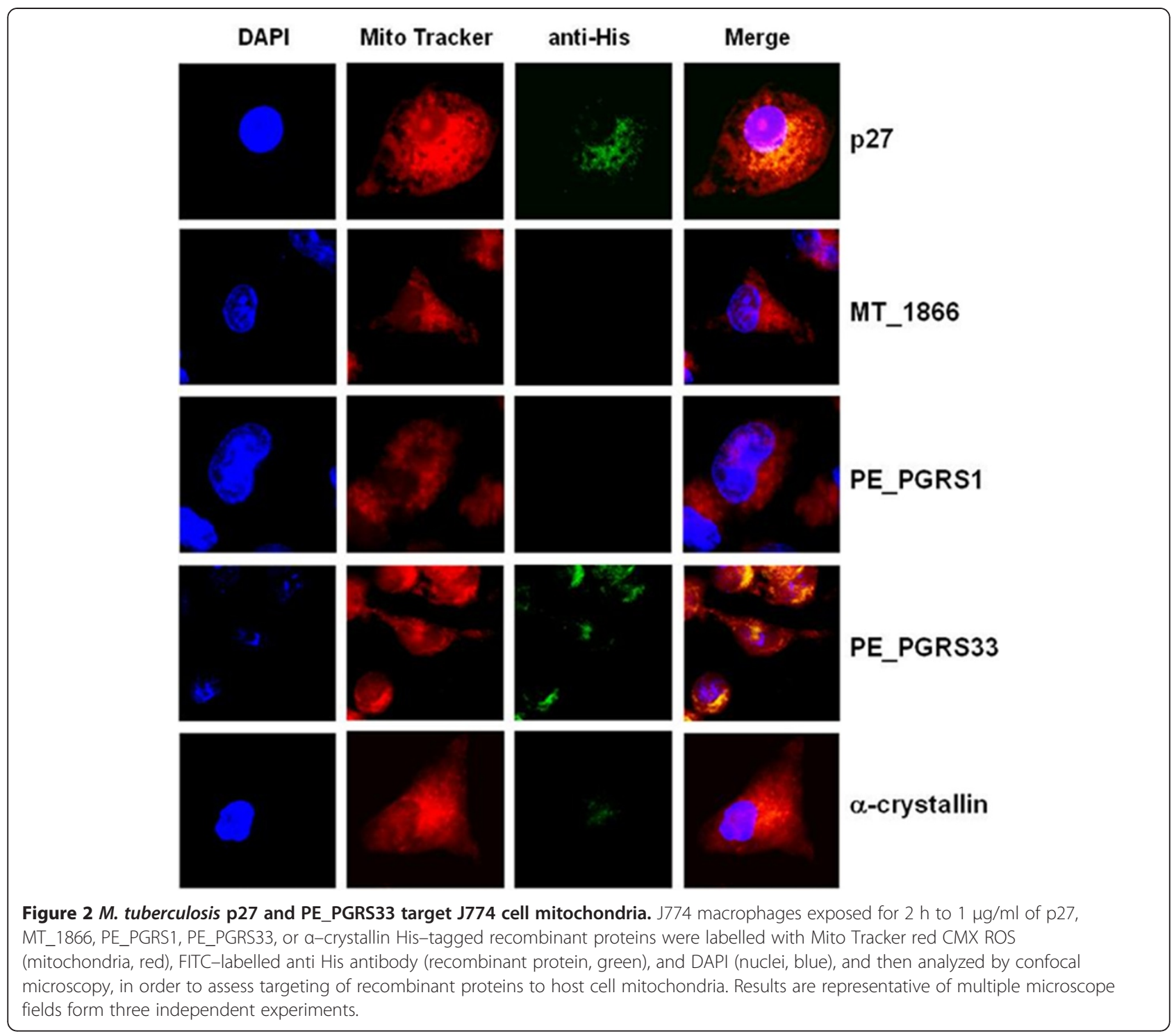

for considering true homology between these two proteins (Table 3).

All in all, our results show, in another experimental system (J774 murine macropheges exposed to the recombinant protein), that PE_PGRS33 targets host cell mitochondria, thus confirming Cadieux et al. [9] findings.

In addition, based on bioinformatic analyses, we present evidence that Rv1411c (p27) which is the M. tuberculosis H37Rv ortholog of M. tuberculosis CDC1551 MT_1455 and a known virulence factor, targets mitochondria (Figure 2).

Identifying other $M$. tuberculosis proteins that target mitochondria would help to clarify the structural requirements for mitochondrial targeting and more important, the role of those proteins in the M. tuberculosis-host cell communication. This work provided a list of 19 candidate proteins, and after experimentally testing some of them and identifying p27 as a mitochondria targeting protein, still leaves some candidate proteins for further analysis.

\section{Conclusions}

Over the past decade it has been shown that in addition to their role in ATP production, mitochondria also function as signal transducers platforms and have an essential role in immune responses and host-pathogen interactions.

In analysing the pathogen-host cell crosstalk, it has been shown that several pathogen proteins target mitochondria thus controlling the host cell fate. Here, we conducted a bioinformatic analysis aimed at identifying $M$. tuberculosis proteins that, based on aminoacid sequence, could possible be secretory, and target mitochondria. 
Our results singled out 19 out of 4, 246 M. tuberculosis predicted proteins as likely to be secretory and harbour mitochondrial targeting sequences. Interestingly, several proteins considered to be virulence factors are included and over-represented in this 19 protein list. Four such proteins were experimentally tested and while confirming that PE_PGRS33 targets mitochondria, a new $M$. tuberculosis mitochondria targeting protein was identified (p27).

This work suggests a correlation between $M$. tuberculosis protein mitochondrial targeting potential and virulence. The interaction between $M$. tuberculosis and its host cell is complex and, as we think, understanding the role of mitochondria in mycobacterial infections and the role of $M$. tuberculosis specific proteins in the crosstalk with host cells at the level of mitochondria will lead us to a better explanation of phenomena such as tuberculosis latency.

\section{Methods}

Bioinformatic analysis of predicted $M$. tuberculosis proteins $M$. tuberculosis predicted protein sequences were downloaded from the $M$. tuberculosis CDC1551 Genome Project from J. Craig Venter Institute/Comprehensive Microbial Resource (JCVI/CMR version 1.0) (ftp://ftp.jcvi. org/pub/data/Microbial_Genomes/m_tuberculosis_cdc1551/ annotation_dbs/m_tuberculosis_cdc1551.pep).

Protein sequences were then analyzed by using the MitoProt II-v1.101 Software (http://ihg.gsf.de/ihg/ mitoprot.html), which calculates the $\mathrm{N}$-terminal protein region that can support a mitochondrial targeting sequence and the cleavage site, as described [34]. M. tuberculosis putative proteins with a probability of at least 0.8500 of having a mitochondrial targeting sequence were selected and re-analyzed by means of the PSORT II prediction algorithm for subcellular localization (http://www.cbs.dtu.dk/services/SignalP-3.0/) which uses the $\mathrm{k}$ nearest neighbours classifier [35]. Those proteins that in addition of scoring well for MitoProt II had the highest probability of being targeted to mitochondria by PSORT II were further analyzed for secretory signal peptides by using SignalP 3.0 (http://www.cbs.dtu.dk/services/ SignalP/), which predicts the presence and location of signal peptide cleavage sites in amino acid sequences from Gram-positive prokaryotes, Gram-negative prokaryotes, and eukaryotes. The method incorporates a prediction of cleavage sites and a signal peptide/non-signal peptide prediction based on a combination of several artificial neural networks and hidden Markov models, as described [42-44].

The amino acid sequences of some proteins likely to harbor mitochondrial targeting sequences, and to be secreted by the CDC1551 strain of M. tuberculosis, were compared against the homologous proteins of
M. tuberculosis H37Rv, M. tuberculosis H37Ra, and M. bovis BCG Pasteur 1173P2 strain, by using the "Protein vs. All Alignment" tool at the JCVI/CMR webpage. The retrieved amino acid sequences were then analyzed by MitoProt II-v1.101, PSORT II, and SignalP 3.0 algorithms as previously described.

\section{M. tuberculosis recombinant proteins}

The cloning, expression and purification of the M. tuberculosis Rv1411c (p27) protein was performed as previously described [45]. The full length of Rv1818c (PE_PGRS33) gene cloned into PET15b fused to a histidine tag was a kind gift from Dr. M.J. Brennan (CBER, FDA, Bethesda, MD, USA). The coding regions for the mycobacterial antigens were amplified by PCR with the high fidelity DNA polymerase $P f x$ (Invitrogen) from $M$. tuberculosis H37Rv genomic DNA (Rv1411c or p27; Rv0109 or PE_PGRS1; Rv1818c or PE_PGRS33) or $M$. tuberculosis CDC1551 (MT_1866) with specific oligonucleotide primers. PCR products were ligated into the pCR4 Blunt-TOPO vector (Invitrogen), and amplified in JM110 Escherichia coli strain (Stratagene) and then subcloned into $\mathrm{pET} 15 \mathrm{~b}$ vector, that was used for the transformation of the E. coli strains Rosetta (DE3) (Novagen) or C41 (DE3) (Avidis S.A.). Expression of the proteins was induced in logarithmic phase cultures. Bacterial pellets were suspended in PBS, sonicated and centrifuged. His-tagged recombinant proteins were purified in an AKTA FLPC system (GE Healthcare), using $1 \mathrm{ml}$ Histrap columns (GE Healthcare). Pooled purified recombinant proteins were dialized against $0.3 \mathrm{M} \mathrm{NaCl}, 20 \mathrm{mM}$ $\mathrm{NaH}_{2} \mathrm{PO}_{4}$, pH 8.0. In addition, the M. tuberculosis Rv2031 protein (also known as $16 \mathrm{kDa}$ antigen, hsp 16.3, hspX, and $\alpha$-crystallin), obtained from E. coli and purified by histidin-affinity chromatography was used as a negative control on the basis of its low probability score for targeting mitochondria (Mi MitoProt II-v1.101, and PSORT II). In all cases, protein concentration was quantified and recombinant proteins were stored at $-70^{\circ} \mathrm{C}$ until use.

\section{Mitochondrial targeting of $M$. tuberculosis recombinant proteins}

J774.A1 murine macrophages were plated on glass coverslips in 6-well culture plates (Corning) at a density of $5 \times 10^{5}$ cells/well and incubated overnight at $37^{\circ} \mathrm{C}$ in $5 \%$ $\mathrm{CO}_{2}$ atmosphere. After washing, cells were incubated in the presence of the indicated $M$. tuberculosis recombinant proteins (p27, MT_1866. PE_PGRS1, PE_PGRS33, or $\alpha-$ crystallin used as a negative control) for $2 \mathrm{~h}$. Cells were then labelled with MitoTracker red CMXRos (Invitrogen) at a final concentration of $100 \mathrm{nM}$, for $30 \mathrm{~min}$ and subsequently fixed and permeabilized (Cytofix/Cytoperm, BD Biosciences) and stained with FITC-labelled anti-His antibody (Miltenyi Biotec). After further washing, cells 
were mounted in DAPI-containing Vectashield (Vector). Imaging was performed in a LSM5 Pascal (Zeiss) confocal microscope, using a 100x oil immersion objective. Online data acquisition and further fluorescence analysis were carried out using LSM5 image browser (Zeiss).

\section{Abbreviations}

M. tuberculosis: Mycobacterium tuberculosis; PE: Prolin-Glutamic acid motif; PPE: Prolin-Prolin-Glutamic acid motif; PGRS: Polymorphic GC-rich repetitive sequence.

\section{Competing interest}

The authors declare no competing interest.

\section{Authors' contributions}

MMBMA conceived the idea, experimental design,analyzed data; ISPG performed experiments; CE provided special reagents, experimental design, analyzed data; MSM performed experiments; RHP provided special reagents, analyzed data; FJSG conceived the idea, experimental design, performed experiments, analyzed data, and wrote the paper. All authors read and approved the final manuscript.

\section{Acknowledgements}

This work was financed in part by a Consejo Nacional de Ciencia y Tecnología grant (CB-2010/158340) and SIP-IPN (20080287), the funders had no role in study design, data collection and analysis, decision to publish, or preparation of the manuscript. We thank Cristina Parada, for assistance on recombinant protein purification, Jacqueline Oliva-Ramírez for help with Confocal microscopy, and Professor Sir Salvador Moncada and Dr. César Hernández-Rodríguez for critical reading of the manuscript. MMBMA and FJSG are COFAA-IPN, EDI, and SNI fellows, CE and RHP are SNI fellows.

\section{Author details}

'Laboratorio de Inmunorregulación, Departamento de Inmunología, Escuela Nacional de Ciencias Biológicas, Instituto Politécnico Nacional, Carpio y Plan de Ayala, Col. Sto. Tomás, México D.F, México. ${ }^{2}$ Departamento de Inmunología, Instituto de Investigaciones Biomédicas, Universidad Nacional Autónoma de México, México D.F, México. ${ }^{3}$ Instituto Nacional de Ciencias Médicas y de la Salud "Salvador Zubirán", México D.F, México.

Received: 6 September 2011 Accepted: 19 December 2012 Published: 22 December 2012

\section{References}

1. WHO: report 2010, http://apps.who.int/iris/bitstream/10665/75938/1/ 9789241564502 eng.pdf.

2. Kenny B, Jepson M: Targeting of an enteropathogenic Escherichia coli (EPEC) effector protein to host mitochondria. Cell Microbiol 2000, 2:579-590.

3. Nougayrede J-P, Donnenberg MS: Enterophatogenic Escherichia coli EspF is targeted to mitochondria and is required to initiate the mitochondrial death pathway. Cell Microbiol 2004, 6:1097-1111.

4. Kozjak-Pavlovic V, Ross K, Rudel T: Import of bacterial pathogenicity factors into mitochondria. Curr Op Microbiol 2008, 11:9-14.

5. Papatheodorou P, Domanska G, Oxle M, Mathieu J, Selchow O, Kenny B, Rassow J: The enterophatogenic Escherichia coli (EPEC) Map effector is imported into the mitochondrial matrix by the TOM/Hsp70 system and alters organelle morphology. Cell Microbiol 2006, 8:677-689.

6. Duan L, Gan H, Golan DE, Remold HG: Critical role of mitochondrial damage in determining outcome of macrophage infection with Mycobacterium tuberculosis. J Immunol 2002, 169:5181-5187.

7. Abarca-Rojano E, Rosas-Medina P, Zamudio-Cortéz P, Mondragón-Flores R, Sánchez-García FJ: Mycobacterium tuberculosis virulence correlates with mitochondrial cytochrome $\mathrm{c}$ release in infected macrophages. Scand I Immunol 2003, 58:419-427.

8. Chen M, Gan H, Remold HG: A mechanism of virulence: virulent Mycobacterium tuberculosis strain H37Rv, but not attenuated H37Ra causes significant mitochondrial inner membrane disruption in macrophages leading to necrosis. J Immunol 2006, 176:3707-3716.
9. Cadieux N, Parra M, Cohen H, Maric D, Morris SL, Brennan MJ: Induction of cell death after localization of the host cell mitocondria by the Mycobacterium tuberculosis PE_PGRS33 protein. Microbiol 2011, 157:793-804.

10. Cole ST, Brosch R, Parkhill J, Garnier T, Churcher C, Harris D, Gordon SV, Eiglmeier K, Gas S, Barry CE 3rd, Tekaia F, Badcock K, Basham D, Brown D, Chillingworth T, Connor R, Davies R, Devlin K, Feltwell T, Gentles S, Hamlin N, Holroyd S, Hornsby T, Jagels K, Krogh A, McLean J, Moule S, Murphy L, Oliver K, Osborne J, Quail MA, Rajandream MA, Rogers J, Rutter S, Seeger K, Skelton J, Squares R, Squares S, Sulston JE, Taylor K, Whitehead S, Barrell BG: Deciphering the biology of Mycobacterium tuberculosis from the complete genome sequence. Nature 1998, 393:537-544.

11. Fleischmann RD, Alland D, Eisen JA, et al: Whole-genome comparison of Mycobacterium tuberculosis clinical and laboratory strains. J Bacteriol 2002, 184:5479-5490.

12. Kapopoulou A, Lew JM, Cole ST: The Mycobrowser portal: a comprehensive and manually annotated resource for Mycobacterial genomes. Tubercul 2010, 91:8-13.

13. Cole ST: Learning from the genome sequence of Mycobacterium tuberculosis H37Rv. FEBS Lett 1999, 452:7-10.

14. Banu S, Honore N, Saint-Joanis B, Philpott D, Prevost M-C, Cole ST: Are the PE_PGRS proteins of Mycobacterium tuberculosis variable surface antigens? Mol Microbiol 2002, 44:9-19.

15. Zheng H, Lu L, Wang B, Pu S, Zhang X, Zhu G, Shi W, Zhang L, Wang H, Wang $S$, Zhao G, Zhang Y: Genetic basis of virulence attenuation revealed by comparative genomic analysis of Mycobacterium tuberculosis strain H37Ra versus H37Rv. PLoS One 2008, 3:e2375.

16. Lucattini R, Likic VA, Lithgow T: Bacterial proteins predisposed for targeting to mitochondria. Mol Biol Evol 2004, 21:652-658.

17. Rohde K, Yates RM, Purdy GE, Russell DG: Mycobacterium tuberculosis and the environment within the phagosome. Immunol Rev 2007, 219:37-54.

18. Armstrong JA, Hart PD: Response of cultured macrophages to Mycobacterium tuberculosis, with observations on fusion of lysosomes with phagosomes. J Exp Med 1971, 134:713-740.

19. Walburger A, Koul A, Ferrari G, Nguyen L, Prescianotto-Baschong C, Huygen K, Kebl B, Thompson C, Bacher G, Pieters J: Protein kinase G from pathogenic mycobacteria promotes survival within macrophages. Science 2004, 304:1800-1804.

20. Axelrod S, Oschkinat H, Enders J, Schlegel B, Brinkmann V, Kaufmann SH, Hass A, Schaible UE: Delay of phagosome maturation by a mycobacterial lipid is reversed by nitric oxide. Cell Microbiol 2008, 10:1530-1545.

21. Ragno S, Romano M, Howell S, Pappin DJ, Jenner PJ, Colston MJ: Changes in gene expression in macrophages infected with Mycobacterium tuberculosis: a combined transcriptomic and proteomic approach Immunol 2001, 104:99-108.

22. Tailleux L, Waddell SJ, Pelizzola M, Mortellaro A, Withers M, Tanne A, Castagnoli PR, Gicquel B, Stoker NG, Butcher PD, Foti M, Neyrolles O: Probing host pathogen cross-talk by transcriptional profiling of both Mycobacterium tuberculosis and infected human dendritic cells and macrophages. PLoS One 2008, 3:e1403.

23. Danelishvili L, McGarvey J, Li YJ, Bermudez LE: Mycobacterium tuberculosis infection causes different levels of apoptosis and necrosis in human macrophages and alveolar epithelial cells. Cell Microbiol 2003, 5:649-660.

24. Kelly DM, ten Bokum AMC, O'Leary SM, O'Sullivan MP, Keane J: Bystander macrophage apoptosis after Mycobacterium tuberculosis H37Ra infection. Infect Immun 2008, 76:351-360.

25. Keane J, Remold HG, Kornfeld H: Virulent Mycobacterium tuberculosis strain evades apoptosis of infected alveolar macrophages. J Immunol 2000, 164:2016-2020.

26. Sly LM, Hingley-Wilson SM, Reiner NE, McMaster WR: Survival of Mycobacterium tuberculosis in host macrophages involves resistance to apoptosis dependent upon induction of antiapoptotic Bcl-2 family member Mcl-1. J Immunol 2003, 170:430-437.

27. Derrick SC, Morris SL: The ESAT6 protein of Mycobacterium tuberculosis induces apoptosis of macrophages by activating caspase expression. Cell Microbiol 2007, 9:1547-1555.

28. Velmurugan $\mathrm{K}$, Chen B, Miller JL, Azogue S, Gurses S, Hsu T, Glickman M, Jacobs WR Jr, Porcelli SA, Briken V: Mycobacterium tuberculosis nuoG is a virulent gene that inhibits apoptosis of infected host cells. PLoS Pathog 2007, 3:e110. 
29. Sanchez A, Espinosa P, Esparza MA, Colon M, Bernal G, Mancilla R: Mycobacterium tuberculosis 38-KDa lipoprotein is apoptogenic for human monocyte-derived macrophages. Scand I Immunol 2009, 69:20-28.

30. Green DR, Kroemer G: The pathophysiology of mitochondrial cell death. Science 2004, 305:626-629.

31. Boya P, Roques B, Kroemer G: New EMBO members/review: viral and bacterial proteins regulating apoptosis at the mitochondrial level. EMBO J 2001, 20:4325-4331.

32. Arnoult D, Carneiro L, Tattoli I, Girardin SE: The role of mitochondria in cellular defense against microbial infection. Semin Immunol 2009, 21:223-232.

33. Gomez M, Johnson S, Gennaro ML: Identification of secreted proteins of Mycobacterium tuberculosis by a bioinformatic approach. Infect Immun 2000, 68:2323-2327.

34. Claros MG, Vincens P: Computational method to predict mitochondrially imported proteins and their targeting sequences. Eur J Biochem 1996, 241:779-786.

35. Horton P, Nakai K: Better prediction of protein cellular localization sites with the k nearest neighbours classifier. Intell Syst Mol Biol 1997, 5:147-152.

36. Leversen NA, de Souza GA, Malen H, Prasad S, Jonassen I, Wiker H: Evaluation of signal peptide prediction algorithms for identification of mycobacterial signal peptides using sequence data from proteomic methods. Microbiol 2009, 155:2375-2383.

37. Abdallah AM, Verboom T, Weerdenburg EM, van Pittius NC G, Mahasha PW, Jiménez C, Parra M, Cadieux N, Brennan MJ, Appelmelk BJ, Bitter W: PPE and PE-PGRS proteins of Mycobacterium marinum are transported via the type VII secretion system ESX-5. Mol Mcrobiol 2009, 73:329-340.

38. Mészáros B, Tóth J, Vértessy BG, Dosztányi Z, Simon I: Proteins with complex architecture as potential targets for drug design: a case study of Mycobacterium tuberculosis. PLoS Comp Biol 2011, 7:e1002118.

39. Espitia C, Laclette JP, Mondragon-Palomino M, Amador A, Campusano J, Martens A, Singh M, Cicero R, Zhang Y, Moreno C: The PE_PGRS glycinerich proteins of Mycobacterium tuberculosis: a new family of fibronectinbinding proteins? Microbiol 1999, 145:3487-3495.

40. Brennan MJ, Delogu G, Chen Y, Bardarov S, Kriakov J, Alavi M, Jacobs WR Jr: Evidence that Mycobacterial PE_PGRS proteins are cell surface constituents that influence interactions with other cells. Infect Immun 2001, 69:7326-7333

41. Blaji KN, Goyal G, Narayana Y, Srinivas M, Chaturvedi R, Mohammad S: Apoptosis triggered by Rv1818c, a PE family gene from mycobacterium tuberculosis is regulated by mitochondrial intermediates in T cells. Microb Infect 2007, 9:271-281.

42. Nielsen H, Engelbrecht J, Brunak S, von Heijne G: Identification of prokaryotic and eukaryotic signal peptides and prediction of their cleavage sites. Prot Engin 1997, 10:1-6.

43. Bendtsen JD, Nielsen H, Von Heijne G, Brunak S: Improved prediction of signal peptides: SignalP 3.0. J Mol Biol 204, 340:783-795.

44. Emanuelsson $\mathrm{O}$, Brunak $\mathrm{S}$, von Heijne $\mathrm{G}$, Nielsen $\mathrm{H}$ : Locating proteins in the cell using TargetP, SignalP and related tools. Nat Protocol 2007, 2:953-971.

45. Gonzalez-Zamorano M, Mendoza-Hernandez G, Xolapa W, Parada C, Vallecillo AJ, Bigi F, Espitia C: Mycobacterium tuberculosis glycoproteomics based on ConA-lectin affinity capture of mannosylated proteins. J Prot Res 2009, 8:721-733.

doi:10.1186/2042-5783-2-9

Cite this article as: Moreno-Altamirano et al:: Bioinformatic identification of Mycobacterium tuberculosis proteins likely to target host cell mitochondria: virulence factors?. Microbial Informatics and Experimentation 2012 2:9.

\section{Submit your next manuscript to BioMed Central and take full advantage of:}

- Convenient online submission

- Thorough peer review

- No space constraints or color figure charges

- Immediate publication on acceptance

- Inclusion in PubMed, CAS, Scopus and Google Scholar

- Research which is freely available for redistribution

Submit your manuscript at www.biomedcentral.com/submit
Ciomed Central 